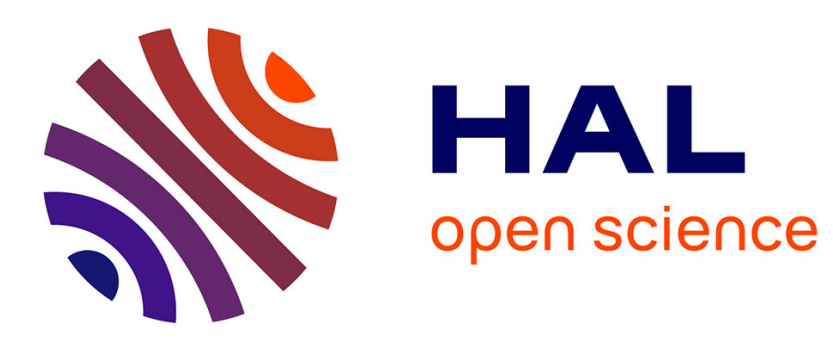

\title{
Atypical beta power fluctuation while listening to an isochronous sequence in dyslexia
}

Andrew Chang, Nathalie Bedoin, Laure-Helene Canette, Sylvie Nozaradan, Dave Thompson, Alexandra Corneyllie, Barbara Tillmann, Laurel J Trainor

\section{- To cite this version:}

Andrew Chang, Nathalie Bedoin, Laure-Helene Canette, Sylvie Nozaradan, Dave Thompson, et al.. Atypical beta power fluctuation while listening to an isochronous sequence in dyslexia. Clinical Neurophysiology, 2021, 10.1016/j.clinph.2021.05.037 . hal-03384362

\section{HAL Id: hal-03384362 \\ https://hal.science/hal-03384362}

Submitted on 18 Oct 2021

HAL is a multi-disciplinary open access archive for the deposit and dissemination of scientific research documents, whether they are published or not. The documents may come from teaching and research institutions in France or abroad, or from public or private research centers.
L'archive ouverte pluridisciplinaire HAL, est destinée au dépôt et à la diffusion de documents scientifiques de niveau recherche, publiés ou non, émanant des établissements d'enseignement et de recherche français ou étrangers, des laboratoires publics ou privés. 


\section{Atypical beta power fluctuation while listening to an isochronous sequence in dyslexia}

3 Andrew Chang ${ }^{1}$, Nathalie Bedoin ${ }^{2,3,4}$, Laure-Helene Canette ${ }^{3,5}$, Sylvie Nozaradan ${ }^{6,7}$, Dave

4 Thompson $^{1,8,9}$, Alexandra Corneyllie ${ }^{3,5}$, Barbara Tillmann $^{3,5, *}$ \& Laurel J. Trainor ${ }^{1,8,9, *}$

$5{ }^{1}$ Department of Psychology, Neuroscience and Behaviour, McMaster University, Hamilton, ON,

6 Canada L8S 4K1

$7 \quad{ }^{2}$ CNRS, UMR5292, INSERM, U1028, Lyon Neuroscience Research Center, IMPACT Team,

8 Bron, France

$9 \quad{ }^{3}$ University Lyon 1, Villeurbanne, France

$10 \quad{ }^{4}$ University Lyon 2, Bron, France

$11{ }^{5}$ CNRS, UMR5292, INSERM, U1028, Lyon Neuroscience Research Center, Auditory Cognition

12 and Psychoacoustics Team, Bron, France

$13{ }^{6}$ The MARCS Institute for Brain, Behaviour and Development, Western Sydney University,

14 Locked Bag 1797, Penrith, NSW, 2751, Australia

$15{ }^{7}$ Institute of Neuroscience (IONS), Université catholique de Louvain (UCL), Avenue Mounier

16 53, Woluwe-Saint-Lambert, 1200, Belgium

$17{ }^{8}$ McMaster Institute for Music and the Mind, McMaster University, Hamilton, ON, Canada L8S

$18 \quad 4 \mathrm{~K} 1$

$19{ }^{9}$ Rotman Research Institute, Baycrest Hospital, Toronto, ON, Canada M6A 2E1

$21{ }^{*}$ Shared last authors and corresponding authors:

22 Laurel J. Trainor, Department of Psychology, Neuroscience and Behaviour, McMaster

23 University, Hamilton, Ontario, Canada L8S 4K1; Phone: 905-525-9140 ext. 23007; Email:

24 ljt@mcmaster.ca. 
25 Barbara Tillmann, Lyon Neuroscience Research Center, Inserm U1028 - CNRS UMR5292 -

26 UCBL. Centre Hospitalier Le Vinatier - Bâtiment 462 - Neurocampus, 95 boulevard Pinel,

2769675 Bron Cedex, France, Email: Barbara.Tillmann@cnrs.fr 


\section{Highlights}

29 - Adults with dyslexia and matched controls listened to isochronous tone sequences.

30 - Dyslexia was associated with an atypical phase of beta $(\sim 20 \mathrm{~Hz})$ power fluctuation.

31 - Atypical beta power fluctuation might reflect deficits in tracking auditory rhythm. 


\section{Abstract}

\section{Objective}

35 Developmental dyslexia is a reading disorder that features difficulties in perceiving and tracking

36 rhythmic regularities in auditory streams, such as speech and music. Studies on typical healthy

37 participants have shown that power fluctuations of neural oscillations in beta band (15-25 $\mathrm{Hz})$,

38 which are likely related to predictive timing and attentional processes, reflect an essential

39 mechanism for tracking rhythm or entrainment. Here we investigate whether adults with dyslexia

40 have atypical beta power fluctuation.

\section{Methods}

43 The electroencephalographic activities of individuals with dyslexia $(n=13)$ and typical control

44 participants $(n=13)$ were measured while they passively listened to an isochronous tone

45 sequence (2 Hz presentation rate). The time-frequency neural activities generated from auditory

46 cortices were analyzed.

$48 \quad$ Results

49 The phase of beta power fluctuation at the $2 \mathrm{~Hz}$ stimulus presentation rate differed and appeared 50 opposite between individuals with dyslexia and controls.

\section{Conclusions}

53 Atypical beta power fluctuation might reflect deficits in perceiving and tracking auditory rhythm 54 in dyslexia. 
57 These findings extend our understanding of atypical neural activities for tracking rhythm in

58 dyslexia and could inspire novel methods to objectively measure the benefits of training, and

59 predict potential benefit of auditory rhythmic rehabilitation programs on an individual basis.

60

61 Keywords: Dyslexia, Electroencephalography (EEG), Beta oscillation, Auditory perception,

62 Entrainment

63

64 Abbreviations: electroencephalography (EEG), event-related potential (ERP), independent 65 component analysis (ICA), standard deviation (SD)

66

67 


\section{Introduction}

Developmental dyslexia is a common reading disorder with a prevalence rate of around 5

to $10 \%$ (Siegel, 2006), featuring impairments in phonological awareness, such as difficulties in

71 identifying rhyming words or syllabic stress patterns in speech (Goswami et al., 2013). Beyond

72 deficits in speech perception, this impairment is also associated with deficits of auditory

73 processing of rhythmic temporal regularity (Goswami, 2011, 2015, 2019), including detecting

74 amplitude envelope onset, perceiving and producing rhythm, and extracting auditory rhythmic

75 regularity (Flaugnacco et al., 2014; Huss et al., 2011; Leong et al., 2011; Leong and Goswami,

76 2014). The association between these two domains of auditory processing are also observed

77 among typically-developing children: rhythm perception and the ability to synchronize to a beat

78 are associated with phonological awareness, verbal short-term memory, rapid naming, and

79 morphosyntactic accuracy in speech production (e.g., Woodruff Carr et al., 2014). Despite this

80 evidence, we know little about the neural processing that underlies the rhythmic deficits in

81 dyslexia, the focus of the present paper.

82 Neural oscillations have been proposed as an essential mechanism for tracking rhythm

83 (Haegens and Zion Golumbic, 2018; Trainor et al., 2018). The power fluctuations of high-

84 frequency oscillations (beta band: $15-25 \mathrm{~Hz}$ ) entrain to rhythmic regularity in sound streams.

85 When participants listen to an isochronous tone sequence, the beta band power from auditory

86 cortex decreases immediately following the onset of a tone, and then rebounds anticipatorily

87 according to the onset time of the upcoming tone (Cirelli et al., 2014; Fujioka et al., 2012),

88 consistent with a mechanism for predicting the onset time of the next expected tone. This

89 entrainment activity can be modulated by hierarchical rhythmic structure or disrupted by a

90 random sequence (Fujioka et al., 2009; 2015; Iversen et al., 2009; Snyder and Large, 2005), and 
91 it is associated with auditory prediction, attentional processing and perceptual performance

92 (Chang et al., 2018, 2019; Morillon and Baillet, 2017). Atypical beta power entrainment

93 activities have been observed in populations featuring deficits in rhythm perception and tracking,

94 such as patients with Parkinson's disease (te Woerd et al., 2017, 2018) and children with

95 stuttering (Etchell et al., 2016). However, it is unknown whether atypical beta power entrainment

96 to auditory rhythmicity is associated with dyslexia.

97 In the present study, we measured the electroencephalographic (EEG) activities of

98 individuals with dyslexia while they passively listened to an isochronous tone sequence. We

99 hypothesized that the beta power fluctuation generated from auditory cortex is different between

100 individuals with dyslexia and typical controls.

101

102 Methods

103 Participants

104 Thirteen adults with developmental dyslexia (seven women, ten right-handed; mean age

$105=23.2$ years, $\mathrm{SD}=2.95$ years) and 13 matched controls (seven women, seven right-handed,

106 mean age $=22.5$ years, $\mathrm{SD}=2.07)$ participated in the current experiment. Although handedness

107 was not perfectly matched in the current study, the higher proportion of non-right-handers

108 appears to be a feature of dyslexia (e.g., Eglinton and Annett, 1994). All participants reported

109 French as their native language, with one dyslexic participant reporting bilingualism with

110 English. None reported auditory deficits. Education levels did not differ between the Dyslexic

111 group $($ mean $=14.92$ years; $S D=1.55)$ and the Control group $($ mean $=15.31$ years, $\mathrm{SD}=0.95)$,

$112 \mathrm{p}=.45$. Musical background, as measured by years of instrumental instruction, also did not 
113 differ between the Dyslexic group (mean $=2.00$ years; $\mathrm{SD}=2.16$ ) and the Control group (mean $114=1.69$ years, $\mathrm{SD}=2.13), \mathrm{p}=0.72$.

115 Participants with dyslexia were part of a larger research project investigating dyslexia at 116 the university level (Abadie and Bedoin, 2016; see Canette et al., 2020, Fiveash et al., 2020, for

117 more information). As detailed in Table S2, they completed a set of language and

118 neuropsychological tests, confirming the persistence of dyslexia for each participant. All

119 participants with dyslexia reported having seen a speech therapist for a dyslexia diagnosis and

120 for training designed to reduce reading difficulties for at least two years (and maximum 5 years)

121 during childhood, which in France is generally defined as between 8 and 13 years old. They

122 mainly have phonological difficulties (i.e., phonological or mixed forms of dyslexia, which are

123 the most frequently observed forms of this neurodevelopmental pathology). Note that all

124 participants with dyslexia had scores in the normal range for nonverbal intelligence (as measured

125 by Raven's Matrices) and they also performed in the normal range for reading comprehension

126 (average $\mathrm{z}$-score placed them above the mean $=1.91, \mathrm{SD}=0.60$ ). None of the participants

127 reported psychiatric or neurological diagnoses or attention disorder with/without hyperactivity.

128 On the other hand, none of the controls reported a history of spoken or written language

129 disorders. In particular we confirmed that, while at school, they did not have any difficulties in

130 language understanding or production or learning to read, and none had been followed up by a 131 speech therapist.

132 Written informed consent based on the French ethics procedure approval Committee

133 (CPP Sud-Est II, 2007-009-3) was obtained from all participants prior to participating in the

134 experiment, which was conducted in accordance with the guidelines of the Declaration of 
135 Helsinki. Participants received a compensation of 12 Euros per hour for their participation in the 136 study.

138 Stimulus

139 During the EEG recording, participants passively listened for 5 minutes to an isochronous 140 sequence of piano tones with an inter-onset interval of $500 \mathrm{~ms}$, which is in the tempo range that 141 gives rise to a strong sense of the beat (Drake et al., 2000; Merchant et al., 2015). The tone was

142 C4 $(262 \mathrm{~Hz})$, from the University of Iowa Musical Instrument Samples, and the amplitude

143 envelope of the piano tone was percussive with $10 \mathrm{~ms}$ rise times. Tones were truncated to be 200

$144 \mathrm{~ms}$ in duration, and a linear decay to zero was applied over the entire excerpt to remove offset

145 artifact (Figure S3). For the behavioural post-test synchronization task, the same tone was

146 played with the same inter-onset interval for either 10 repetitions (training trial) or 40 repetitions

147 (experimental trials).

\section{Procedure}

150 To keep participants awake and still, they watched a silent black and white movie with

151 Charlie Chaplin while they listened passively to the 5-minute isochronous tone sequence (and

152 two other rhythmic patterns, not presented here) while EEG was continuously recorded ${ }^{1}$. This

153 EEG recording was part of a longer experimental session and followed a priming experiment

154 with an active task (reported in Canette et al., 2020 and Fiveash et al., 2020). After the EEG

155 recording session, participants came back to the laboratory for a second testing session and

\footnotetext{
${ }^{1}$ It is a common approach to have participants watch a silent movie during a passive listening task to keep them awake and reduce eyeblinks or eye movements during EEG/MEG recordings. As the movie is not time-locked to the tone sequence and is not rhythmic, its influence on EEG analyses is negligible (e.g., Kong et al., 2014; Luo \& Ding, 2020).
} 
156 completed a set of behavioural tests on syntax processing and rhythm perception and production

157 based on the complex Beat Alignment Task (Einarson and Trainor, 2015; data presented in

158 Canette et al., 2020) and two rhythmic patterns (except for one dyslexic participant who did not

159 come back to this second session); these data were presented in Canette et al. (2020). In addition,

160 relevant for the present experiment, participants performed a production task where they were

161 required to tap along to the excerpts of the isochronous sequence used in the EEG session (i.e.,

162 inter-tone-onsets of $500 \mathrm{~ms}$ ). Participants drummed with a stick on a drum pad (Roland, V-

163 Drums) to each tone. Both the passive listening and production parts were implemented with the

164 software Presentation (Neurobehavioural Systems), and stimuli were presented over headphones

165 (Pioneer, HDJ-500). During a training trial, they tapped to a sequence of 10 tones. Following

166 this, they completed two trials of 40 tones (i.e., $20 \mathrm{~s}$ duration) each.

\section{EEG recording and preprocessing}

The EEG signal was recorded with $95 \mathrm{Ag} / \mathrm{AgCl}$ active electrodes (actiCAP 96Ch

170 Standard-2, Brain Products $\mathrm{GmbH}$ ). The signal was recorded with a BrainAmp amplifier at a

171 resolution of 16 bits, a sampling rate of $500 \mathrm{~Hz}$, and with an analog low pass filter of $1000 \mathrm{~Hz}$

172 and high pass filter of $0.016 \mathrm{~Hz}$. The ground electrode was placed at position AFz, the reference

173 electrode on the tip of the nose, and an eye-movement monitoring electrode under the right eye

174 at position Iz. Electrode impedances were kept below $20 \mathrm{k} \Omega$, which is a well acceptable

175 impedance levels for EEG recordings (e.g., Ferree et al., 2001; Luck, 2014). Continuous EEG

176 data were transformed to an average reference offline. The EEG data was further processed in

177 MATLAB with the FieldTrip toolbox (RRID: SCR_004849) (Oostenveld et al., 2011). 
Independent component analysis (ICA) was used to remove artifact signals (Jung et al.,

179 2000). To speed up the ICA performance, the continuous recordings were downsampled to 250

$180 \mathrm{~Hz}$, filtered between 0.7 and $83 \mathrm{~Hz}$ and then segmented into time window from -3 to $303 \mathrm{~s}$ time-

181 locked to the first tone of the sequence. This window covered the entire stimulus sequence

182 length. We went back to the unfiltered data, projected it to ICA space and the ICs reflecting

183 artifact (identified by visual inspection, range of 2-13 ICs), including eye blinking, eye

184 movement, electrocardiogram, and powerline noise, were excluded, and then the data in the ICA

185 space was projected back to 95-channel space for all subsequent analyses. ${ }^{2}$

\section{Modeling dipole sources for auditory cortex}

In the present study, we focused on the responses generated from auditory cortex, as prior

189 studies have shown that auditory beta oscillations are related to temporal prediction and rhythmic

190 tracking (Chang et al., 2018, 2019; Cirelli et al., 2014; Fujioka et al., 2012, 2015). To achieve

191 this goal, we used a dipole model as a spatial filter to largely separate the activities of auditory

192 cortex from other sources, following the studies cited above, which is preferred to analyzing data

193 on selected surface electrodes, as each surface electrode reflects a mixture of activities from

194 many brain sources. The P1 ERP component ( 60-90 ms) was used to localize bilateral auditory

195 cortices, with the dipole locations (but not orientation) constrained to be bilaterally symmetric.

\footnotetext{
${ }^{2}$ Although the electromagnetic field of the headphones was not masked, which might lead to some contamination of EEG recordings from sound waveforms, any potential headphone artifact should have had a negligible influence on our beta band findings because: (1) the observed beta power fluctuation shape was similar to those of previous studies (see Power spectrum of beta power time series); (2) we observed group differences (while a potential artefact would have equally affected recordings of each participant); and (3) this potential artifact was most likely attenuated by the ICA procedure. A potential artifact might have had more influence on early ERP analyses (reported in the Supplementary Materials), which showed somewhat atypical scalp topography in comparison with previous studies.
} 
196 We used the multiple source probe scan algorithm and the four-shell ellipsoid model included in 197 the Brain Electrical Source Analysis (BESA, RRID: SCR_009530) software package. We chose 198 P1 because (1) it is primarily generated from primary auditory cortex (Godey et al., 2001), (2) it

199 is the dominant peak at fast presentation rates while the N1 peak is strongly reduced at fast rates 200 relevant to the current study (Näätänen and Picton, 1987), and (3) previous studies with similar 201 experimental designs also used P1 for localizing auditory cortex (e.g., Fujioka et al., 2012). The 202 mean locations (averaged across participants) of the fitted dipoles were $[ \pm 44.23,-1.95,7.26]$

203 (Talairach coordinates) with approximate mean orientations $[0.2,0.5,0.8]$ (left) and $[-0.2,0.6$, 204 0.8] (right). These locations are close to bilateral primary auditory cortices with orientations 205 toward the mid-frontal surface area, consistent with typical auditory evoked potentials. The mean 206 residual variances of the source fittings were $7.9 \%$ (range 3.4 to 16.3\%) for the Control group 207 and $7.0 \%$ (range 2.4 to $15.4 \%$ ) for the Dyslexia group, and the residual variances were not 208 different between groups $(\mathrm{t}(24)=0.67, \mathrm{p}=0.509)$. Finally, the unfiltered continuous 95-channel 209 EEG was projected into source-space EEG via the dipole model for further time-frequency 210 analyses.

211 Note that it is important that we perform the analyses at dipole locations, as the MEG 212 study of Fujioka et al. (2012) reported that the beta power generated in motor regions fluctuated 213 at the opposite phase of the beta power in auditory cortex. As the spatial resolution of EEG is 214 less precise than MEG, these two signals are likely to be mixed and potentially cancel each other 215 in EEG analyzed from the surface channels. 
218 The unfiltered continuous source-space EEG data was segmented to include non-

219 overlapping epochs of 20 tones each. Each epoch was thus $11 \mathrm{~s}$ long, including $0.5 \mathrm{~s}$ prior to the

220 first tone and $0.5 \mathrm{~s}$ following the twentieth tone as buffer periods to avoid edge artifacts for the

221 time-frequency analysis. The first epoch at the beginning of each recording was excluded from

222 further analysis, as the rhythmic temporal regularity might not yet be perceived. Subsequently,

223 epochs were excluded that were greater than $150 \mu \mathrm{V}$ to ensure artifacts were eliminated that were

224 not removed by ICA. The mean number of usable trials was 23.8 in the Control group and 26.7

225 in Dyslexia group; this was not significantly different between groups $(t(24)=1.20, p=0.244)$.

226 We focused on the induced (non-phase-locked) power in beta band, following our

227 previous studies (e.g., Fujioka et al., 2012; Chang et al., 2016a, 2018, 2019; Cirelli et al., 2014).

228 For each participant, the unfiltered mean ERP waveform (averaged across trials) was subtracted

229 from each epoch to obtain induced waveforms (Fujioka et al., 2012; Cohen, 2014). We used a

230 short-time Fourier transformation with overlapped time windows (moving successively by one

231 sample) to perform the time-frequency transformation for $15-25 \mathrm{~Hz}$ (frequency bin size $=1 \mathrm{~Hz}$ ).

232 The window size was fixed at $500 \mathrm{~ms}$ and a Hanning taper was applied. For each frequency bin,

233 the power was baseline corrected (percentage change) to the mean power for the $0-10 \mathrm{~s}$ window,

234 then the beta power was obtained by averaging the power across $15-25 \mathrm{~Hz}$ bins. To further

235 analyze the fluctuation of the beta power time series, we performed another Fourier

236 transformation with a Hanning taper for the beta power time series for each $10 \mathrm{~s}$ epoch (single-

237 trial), excluding the $0.5 \mathrm{~s}$ buffer periods at both ends.

238 Although some studies have defined the range of beta band to be as wide as $13-30 \mathrm{~Hz}$, we

239 defined it as $15-25 \mathrm{~Hz}$ for several reasons. (1) It is consistent with our previous studies (e.g., 
240 Fujioka et al., 2012; Chang et al., 2019). (2) Some other studies have interpreted activities below

$24115 \mathrm{~Hz}$ as alpha band (e.g., Ahveninen et al., 2017) and above $25 \mathrm{~Hz}$ as low-gamma band (e.g.,

242 Giroud et al., 2020). Therefore, it is debatable whether to include these frequencies as beta band.

243 (3) Considering the imprecision of time-frequency analysis in terms of frequency resolution,

244 activities closer to 13 or $30 \mathrm{~Hz}$ will be more likely contaminated by activities below 13 or above

$24530 \mathrm{~Hz}$, respectively, compared to frequencies between 15 and $25 \mathrm{~Hz}$.

246 For completeness, we also analyzed event-related potentials (ERPs) (see Supplementary

247 Materials).

Tapping production task

250 We used circular statistics (Berens, 2009; Dalla Bella and Sowinski, 2015) to analyze the 251 production performance of the synchronization task for isochronous sequences, leading to two

252 measures related to the resulting vector R: its angle, representing synchronization accuracy and 253 its length, representing synchronization consistency (ranging from 0 to 1, with 1 representing 254 perfect consistency).

256 Statistics

257 We used a nonparametric cluster-based permutation test to analyze the EEG measure 258 difference between groups with the FieldTrip toolbox (Oostenveld et al., 2011). In short, this 259 procedure reduces the number of multiple comparisons by testing the statistical difference at the 260 level of time and/or frequency clusters instead of individual time and/or frequency points, and it 261 has been widely used for analyzing EEG and MEG data (see Maris and Oostenveld, 2007 for 262 more details). Specifically, first we performed a two-sample t-test between the two groups 
263 (Dyslexia, Control) for each time or frequency sample as the initial scan for clustering. Second, 264 we grouped adjacent frequency (Figure 1B) or time (Figure S1A and S1C) samples reaching a

265 threshold of $p<0.05$ in the initial scan into single clusters and summed the t-values of all

266 samples within each cluster as a cluster-level statistic. Third, we built a null distribution with

2671000 random iterations. In each iteration, we pooled all 26 participants (13 in Dyslexia group

268 and 13 in Control group), randomly split participants into 2 equal-size groups, and performed the

269 same calculations as in the first two steps. The null distribution was composed by the summed t-

270 values of the largest suprathreshold cluster of each iteration, and the final p-value was obtained

271 by comparing the observed clustered summed t-value relative to the null distribution. In sum, if

272 there was no group difference, the cluster(s) of the observed data should resemble the random-

273 split data of the null distribution. The final p-value was corrected to two-tailed.

274 The circular statistics were computed in the Circular Statistics Toolbox for MATLAB

275 (Berens, 2009). Participant group differences for $R$ angle and length were assessed with two276 tailed independent tests.

277 Note that the Watson-Williams test assumes the data to be distributed according to a von

278 Mises distribution, i.e., with concentration parameter $(\kappa)$ equal or above 1 . Therefore, we

279 examined whether our $2 \mathrm{~Hz}$ beta power fluctuation time series met this assumption. The $\kappa$

280 parameter was similar between compared datasets (difference range: 0.05-0.14), with only $\kappa$

281 parameters of the left and right auditory cortex of the Control group above 1. Although our data

282 did not fully satisfy the assumptions, the Watson-Williams test is considered robust against

283 deviations from these assumptions (Berens, 2009). 


\section{Results}

286 Power spectrum of beta power time series

The beta power time series of left and right auditory cortex are visualized in Figure 1A.

288 The Control group showed typical beta power fluctuations in that the peaks occurred at the

289 approximate times of tone onsets (with anticipatory increase in power prior to tone onsets) and

290 troughs between tones. This pattern is similar to those observed in previous studies (e.g., Chang

291 et al., 2019; Fujioka et al., 2012, 2015). In contrast, the beta power fluctuations appear atypical

292 in the Dyslexia group in that troughs occurred at the approximate times of tone onsets and peaks

293 occurred between tones.

Figure 1

transformed the beta power time series into a power spectrum for each participant. The spectra of

299 beta power fluctuations (note that this is not the spectra of raw EEG waveform) are shown in

300 Figure 1B. The cluster-based permutation tests on left or right auditory cortex did not show any

301 significant differences in the power spectrum of beta power fluctuations between the Dyslexia

302 and Control groups (range of power fluctuations 1-5 Hz). There was also no interaction between

303 left/right auditory cortex and groups.

The frequency spectrum of beta power fluctuation (Figure 1B) did not show an obvious

305 peak at $2 \mathrm{~Hz}$ as expected, despite the appearance of a power fluctuation around the $2 \mathrm{~Hz}$ tone

306 onset rate in the averaged time domain waveforms (Figure 1A). Nevertheless, we speculated that

307 the beta power fluctuations likely differed in phase between the two groups, and we extracted the 
308 phase of the beta power time series at $2 \mathrm{~Hz}$ (the stimulus presentation rate) from the frequency

309 transformation above (Figure 1C) for subsequent exploratory analyses. Note that the phase of

310 beta power time series refers to the peak/trough position of the fluctuations in the measured beta

311 power relative to the onsets of the stimuli. A Watson-Williams two-sample test showed that the

312 phase of the beta power time series differed significantly at right auditory cortex $(F(1,24)=$

$31312.90, \mathrm{p}=.002$ ), but not at left auditory cortex, although there was a trend for a difference

$314(\mathrm{~F}(1,24)=3.51, \mathrm{p}=.073)$. The interaction between groups and left/right auditory cortex (taking

315 the circular difference between hemispheres within each participant, and then performing a

316 between-subject Watson-Williams test) was not significant $(F(1,24)=1.57, p=.222)$.

317 To investigate whether this significant right auditory cortex phase difference of beta

318 power fluctuation between groups was specific to $2 \mathrm{~Hz}$, we further performed the same analyses

319 at 1 and $3 \mathrm{~Hz}$ in the right auditory cortex. At $3 \mathrm{~Hz}$, there was no significant difference between

320 groups, $\mathrm{F}(1,24)=0.88, \mathrm{p}=.356$. Further, the group difference was larger at $2 \mathrm{~Hz}$ than at $3 \mathrm{~Hz}$

321 (taking the circular difference between frequencies within each participant, and then performing

322 a between-subject Watson-Williams test), $\mathrm{F}(1,24)=9.47, \mathrm{p}=.005$. Thus, the phase differences

323 between groups were markedly larger at $2 \mathrm{~Hz}$ than at $3 \mathrm{~Hz}$. At $1 \mathrm{~Hz}$, the group difference was not

324 significant, but there was a trend, $F(1,24)=3.33, p=.081$. There was also a trend for larger

325 group difference at $2 \mathrm{~Hz}$ than at $1 \mathrm{~Hz}, \mathrm{~F}(1,24)=3.37, \mathrm{p}=.079$. Together these results suggest

326 that the phase difference of beta band power fluctuation is likely specific to $2 \mathrm{~Hz}$, the rate of tone

327 presentation in the stimulus. 
The two participant groups differed in neither synchronization consistency (vector length;

$331 \mathrm{t}(23)=-0.10, \mathrm{p}=.93$ ) nor synchronization accuracy (vector angle; Watson-Williams two-sample

332 test: $\mathrm{F}(1,23)=0.00, \mathrm{p}=.99)$. Both control and dyslexic participants performed well on the

333 tapping task, reaching synchronization consistency (vector length) close to one (controls: .95

$334 \pm .05$; dyslexics: $95 \pm .04$ ) and synchronization accuracy (vector angle) suggesting weak

335 anticipation of the next tone (controls: $-11 \mathrm{~ms} \pm 13$; dyslexics: $-15 \mathrm{~ms} \pm 39$ ).

\section{Discussion}

The results of the current study revealed that the phase of beta power fluctuation was

339 different between adult university students with dyslexia and typical controls while listening to

340 an isochronous tone sequence. To the best of our knowledge, this is the first study reporting

341 atypical beta power entrainment in dyslexia.

342 The atypical beta power fluctuation in dyslexia might reflect deficits in tracking and/or

343 perceiving auditory rhythms. Previous studies on typical healthy participants showed that the

344 fluctuation of beta power reflects entrainment to the rhythmic regularity of an auditory sequence.

345 Specifically, the peaks of the beta power align to the onset of the tones, and are associated with

346 rhythm tracking and auditory perception and prediction (Chang et al., 2018, 2019; Cirelli et al.,

347 2014; Fujioka et al., 2009, 2012, 2015; Snyder and Large, 2005; Iversen et al., 2009). Our neural

348 findings are consistent with previous studies showing that individuals with dyslexia have deficits

349 in perceiving the rhythmic modulation of nonverbal auditory streams and speech (Goswami et

350 al., 2002; Goswami, 2011, 2015, 2019; Megnin- Viggars and Goswami, 2013). Nevertheless, it

351 is important to note that we cannot eliminate all explanations based on factors beyond 
352 entrainment, such as, for example, that the participants in the Dyslexic group were more

353 distracted by the visual movie, leading to less attention to the auditory stimuli.

354 It was unexpected that the frequency spectrum of beta power fluctuation (Figure 1B) did

355 not show an obvious peak the at stimulus presentation rate $(2 \mathrm{~Hz})$. To the best of our knowledge,

356 our study is the first one reporting the spectrum of beta power fluctuation, while most previous

357 studies only reported its time-domain features. The lack of an obvious spectral peak could simply

358 be due to a smaller signal-to-noise ratio in the current study, as the number of trials was smaller

359 and the EEG recording time was shorter than in previous studies (e.g., Fujioka et al., 2012). It is

360 also possible that the power fluctuation was not perfectly periodic; the moment-to-moment

361 fluctuation rate might have drifted around $2 \mathrm{~Hz}$ on individual trials, and thus the $2 \mathrm{~Hz}$ peak was

362 not obvious in the averaged spectrum with traditional Fourier-based time-frequency

363 transformation approaches, which assume the signals to be stable over time. Future studies using

364 more advanced cycle-by-cycle analysis approaches (e.g., Cole and Voytek, 2019) are needed to

365 investigate the dynamic beta oscillatory activities.

366 There are a few studies reporting an association between beta oscillations and dyslexia. A

367 previous study reported that auditory steady-state response synchronization is reduced in the beta

368 frequency range in dyslexia, and that this response is associated with literacy skills (Van Hirtum

369 et al., 2019). Differences in overall beta power at occipital channels between individuals with

370 and without dyslexia have also been found during listening to noise-vocoded speech (Power et

371 al., 2016). An atypical hemispheric asymmetry of beta power in response to linguistic tasks has

372 also been associated with reading skill or processing phonological information in dyslexia (e.g.,

373 Penolazzi et al., 2010; Spironelli et al., 2008). The present study extends these studies by 
374 indicating that the beta oscillatory activity in response to a simple, rhythmic (isochronous) sound 375 sequence is also atypical in dyslexia.

376 Atypical beta power fluctuation has also been observed in other disorders featuring

377 deficits in perceiving or tracking auditory rhythm. For example, Parkinson's patients are known

378 to have deficits in tracking auditory rhythms (Grahn and Brett, 2009; Grahn, 2012), and the

379 phase of their beta power fluctuation while perceiving auditory or visual rhythms was opposite to 380 that of the controls (te Woerd et al., 2017, 2018). Also, children with stuttering have deficits in

381 perceiving auditory rhythm (Chang et al., 2016b; Falk et al., 2015; Wieland et al., 2015), and the 382 phase of their beta power fluctuation was also opposite to that of controls (Etchell et al., 2016). Although previous studies investigating children with dyslexia showed deficits in their

384 ability to tap to a beat or perceive a beat (Muneaux et al., 2004, Thomson and Goswami, 2008), 385 in the present study, performance on a simple metronome tapping task did not differ between 386 adults with dyslexia and typical controls, and we failed to find any associations between atypical 387 beta power fluctuation and tapping production performance to the same isochronous sequence 388 (Figure S2). In addition to the relatively small sample size and thus potentially insufficient 389 statistical power, it is possible that (1) our task was too easy and thus not sensitive enough to 390 reflect a deficit in rhythm processing in dyslexia. Indeed, in another study, our participants with 391 dyslexia were worse than controls (Canette et al., 2020) on performing more complex tapping 392 task (e.g., tapping to the beat of musical excerpts). Furthermore, (2) individuals with dyslexia 393 might find other ways and use different neural mechanisms to compensate as they get older (cf.

394 Shaywitz et al., 2003; Law et al., 2015), making it challenging to directly associate beta 395 oscillation with rhythmic abilities. In particular, previous behavioral studies have revealed 396 greater deficits in tapping tasks for dyslexic adolescents than for dyslexic university students, 
397 who performed as well as controls on some of the measures (Wolff et al., 1990; see also

398 Thomson et al., 2006). Furthermore, the observed variability of the dependent measures of the

399 participants with dyslexia in our study suggests that individuals might use different neural

400 mechanisms.

401 In additional analyses examining ERPs (presented in the Supplementary materials),

402 another common approach to examining auditory neural signature of dyslexia, we found a group

403 difference in an early ERP component at the P1 latency at Fpz, and a marginal correlation

404 between this component and the phase of beta power fluctuation (Figure S1). A possible post hoc

405 explanation is that the atypical beta power fluctuation in dyslexia is associated with deficits in

406 early auditory processing, such as encoding rise time and duration (Bamiou et al., 2001;

407 Hämäläinen et al., 2013; Sharma et al., 2009; King et al., 2003). However, our study was not

408 designed to examine this possibility, so future studies are needed with a larger sample size to

409 investigate this possibility.

410 The current study did not investigate delta $(1-3 \mathrm{~Hz})$ oscillations or their cross-frequency

411 coupling with beta oscillations, despite previously reported associations between delta

412 oscillations and dyslexia. Both animal electrophysiology and human neuroimaging studies have

413 shown that the delta oscillation phase time-locks and entrains to external rhythmic sensory input

414 (see Haegens and Zion Golumbic, 2018 for a review) and it has been reported that atypical delta

415 phase entrainment is associated with deficits of rhythm perception and tracking among

416 individuals with dyslexia (Colling et al., 2017; Di Liberto et al., 2018; Molinaro et al., 2016;

417 Power et al., 2013, 2016). However, recent studies have shown that it is challenging to reliably

418 quantify delta phase entrainment because the frequency-domain signature of ERP activity

419 typically overlaps with low-frequency oscillations in Fourier-based analysis (Doelling et al., 
420 2019; Haegens and Zion Golumbic, 2018). For this reason, we have left the question of how

421 delta and beta oscillations are related in dyslexia to future studies with materials and designs

422 more appropriate for addressing this question. Note that the induced beta oscillations measured

423 in the current study are unlikely to be confounded with ERPs because (1) the mean spectrum of

424 the ERP showed that the power above $15 \mathrm{~Hz}$ was neglectable (Figure S4), and (2) we removed

425 the phase-locked evoked activity from the induced activity in the beta band prior to conducting 426 our analyses.

427 Understanding atypical beta power entrainment could potentially benefit individuals with

428 dyslexia. Studies have shown that rhythmic auditory or musical training can improve the

429 phonological awareness and reading skills in children with dyslexia, including randomized

430 control trials (e.g., Cogo-Moreira et al., 2013; Flaugnacco et al., 2015; Habib et al., 2016;

431 Thomson et al., 2013). The efficacy of longer-term musical rhythm training in language

432 remediation is likely related to the importance of rhythm for decoding the speech stream

433 (Flaugnacco et al., 2015; Kotz and Schwartze, 2010; Overy, 2000; Schön and Tillmann, 2015).

434 In typically-developing children and in children with language disorders, presenting a rhythmic

435 prime has been shown to immediately improve syntactic processing over the short term (Bedoin

436 et al., 2016; Chern et al., 2018; Przybylski et al., 2013). Future studies are needed to investigate

437 whether beta power entrainment is an underlying neural mechanism of these effects, and whether

438 individual differences in beta power entrainment could predict the potential benefit of auditory

439 rhythmic habilitation programs. 


\section{Acknowledgements}

442 We thank Raphaëlle Abadie for her help in the neuropsychological testing of the dyslexic

443 participants. This research was supported by grants from ANR to BT and NB (Grant Agreement

444 number ANR-16-CE28-0012), grants to LJT from the Canadian Institutes of Health Research

445 (MOP 153130) and the Canadian Institute for Advanced Research, a visiting exchange

446 fellowship to LJT from the Erasmus Mundus Auditory Cognitive Neuroscience network, grant to

447 SN from the ERC (ERC Starting Grant 2018 number 801872 Rhythm and Brains), and a Vanier

448 Canada Graduate Scholarship to AC. The team "Auditory cognition and psychoacoustics" is part

449 of the LabEx CeLyA ("Centre Lyonnais d'Acoustique", ANR-10-LABX-60), and L-HC is

450 supported by a post-doctoral grant of the LabEx CeLyA.

451

\section{Conflict of Interest Statement}

453 None

454

455 References

456 Abadie, R., \& Bedoin, N. (2016). Les étudiants dyslexiques à l’Université : Quels déficits

457 cognitifs et langagiers? Neurologies, 19, 298-303.

458 Ahveninen, J., Seidman, L. J., Chang, W. T., Hämäläinen, M., \& Huang, S. (2017). Suppression

459 of irrelevant sounds during auditory working memory. NeuroImage, 161, 1-8.

460 Bamiou, D. E., Musiek, F. E., \& Luxon, L. M. (2001). Aetiology and clinical presentations of 461 auditory processing disorders - a review. Arch Dis Child, 85, 361-365. 
462

463

464

465

466

467

468

469

470

471

472

473

474

475

476

477

478

479

480

481

482

483

484

Bedoin, N., Brisseau, L., Molinier, P., Roch, D., \& Tillmann, B. (2016). Temporally regular musical primes facilitate subsequent syntax processing in children with specific language impairment. Front Neurosci, 10, 245.

Berens, P. (2009). CircStat: A MATLAB toolbox for circular statistics. J Stat Softw, 31, 1-21.

Canette, L.-H., Fiveash, A., Krzonowski, J., Corneyllie, A., Lalitte, P., Thompson, D., Trainor, L., Bedoin, N., \& Tillmann, B. (2020). Regular rhythmic primes boost P600 in grammatical error processing in dyslexic adults and matched controls. Neuropsychologia, $138,107324$.

Chang, A., Bosnyak, D. J., \& Trainor, L. J. (2016a). Unpredicted pitch modulates beta oscillatory power during rhythmic entrainment to a tone sequence. Front Psychol, 7, 327.

Chang, A., Bosnyak, D. J., \& Trainor, L. J. (2018). Beta oscillatory power modulation reflects the predictability of pitch change. Cortex, 106, 248-260

Chang, A., Bosnyak, D. J., \& Trainor, L. J. (2019). Rhythmicity facilitates pitch discrimination: Differential roles of low and high frequency neural oscillations. NeuroImage, 198, 31-43.

Chang, S. E., Chow, H. M., Wieland, E. A., \& McAuley, J. D. (2016b). Relation between functional connectivity and rhythm discrimination in children who do and do not stutter. NeuroImage-Clin, 12, 442-450.

Chern, A., Tillmann, B., Vaughan, C., \& Gordon, R. L. (2018). New evidence of a rhythmic priming effect that enhances grammaticality judgments in children. J Exp Child Psychol, $173,371-379$.

Cirelli, L. K., Bosnyak, D., Manning, F. C., Spinelli, C., Marie, C., Fujioka, T., ... \& Trainor, L. J. (2014). Beat-induced fluctuations in auditory cortical beta-band activity: using EEG to measure age-related changes. Front Psychol, 5, 742. 
Cogo-Moreira, H., de Avila, C. R. B., Ploubidis, G. B., \& de Jesus Mari, J. (2013). Effectiveness of music education for the improvement of reading skills and academic achievement in young poor readers: a pragmatic cluster-randomized, controlled clinical trial. PLOS One, $8(3)$.

Cohen, M. X. (2014). Analyzing neural time series data: theory and practice. MIT press.

Cole, S., \& Voytek, B. (2019). Cycle-by-cycle analysis of neural oscillations. J Neurophysiol, $122,849-861$.

Colling, L. J., Noble, H. L., \& Goswami, U. (2017). Neural entrainment and sensorimotor synchronization to the beat in children with developmental dyslexia: An EEG study. Front Neurosci, 11, 360.

Dalla Bella, S., \& Sowiński, J. (2015). Uncovering beat deafness: detecting rhythm disorders with synchronized finger tapping and perceptual timing tasks. JoVE-J Vis Exp, 97, e51761.

Di Liberto, G. M., Peter, V., Kalashnikova, M., Goswami, U., Burnham, D., \& Lalor, E. C. (2018). Atypical cortical entrainment to speech in the right hemisphere underpins phonemic deficits in dyslexia. NeuroImage, 175, 70-79.

Doelling, K. B., Assaneo, M. F., Bevilacqua, D., Pesaran, B., \& Poeppel, D. (2019). An oscillator model better predicts cortical entrainment to music. P Natl Acad Sci USA, 116(20), 10113-10121.

Drake, C., Jones, M. R., \& Baruch, C. (2000). The development of rhythmic attending in auditory sequences: attunement, referent period, focal attending. Cognition, 77(3), 251-288.

Eglinton, E., \& Annett, M. (1994). Handedness and dyslexia: A meta-analysis. Perceptual and motor skills, 79(3_suppl), 1611-1616. 
Etchell, A. C., Ryan, M., Martin, E., Johnson, B. W., \& Sowman, P. F. (2016). Abnormal time course of low beta modulation in non-fluent preschool children: a magnetoencephalographic study of rhythm tracking. NeuroImage, 125, 953-963.

Falk, S., Müller, T., \& Dalla Bella, S. (2015). Non-verbal sensorimotor timing deficits in children and adolescents who stutter. Front Psychol, 6, 847.

Ferree, T. C., Luu, P., Russell, G. S., \& Tucker, D. M. (2001). Scalp electrode impedance, infection risk, and EEG data quality. Clin Neurophysiol, 112, 536-544.

Fiveash, A., Schön, D., Canette, L.-H., Morillon, B., Bedoin, N., \& Tillmann, B. (2020). A stimulus-brain coupling analysis of regular and irregular rhythms in adults with dyslexia and controls. Brain Cognition, 140. doi: 10.1016/j.bandc.2020.105531

Flaugnacco, E., Lopez, L., Terribili, C., Montico, M., Zoia, S., \& Schön, D. (2015). Music training increases phonological awareness and reading skills in developmental dyslexia: A randomized control trial. PLOS One, 10(9), e0138715.

Flaugnacco, E., Lopez, L., Terribili, C., Zoia, S., Buda, S., Tilli, S., Monasta, L, Montico, M., Sila, A., Ronfani, L., \& Schön, D. (2014). Rhythm perception and production predict reading abilities in developmental dyslexia. Front Human Neurosci, 8, 392.

Fujioka, T., Ross, B., \& Trainor, L. J. (2015). Beta-band oscillations represent auditory beat and its metrical hierarchy in perception and imagery. J Neurosci, 35(45), 15187-15198.

Fujioka, T., Trainor, L. J., Large, E. W., \& Ross, B. (2012). Internalized timing of isochronous sounds is represented in neuromagnetic beta oscillations. J Neurosci, 32(5), 1791-1802.

Fujioka, T., Trainor, L., Large, E., \& Ross, B. (2009). Beta and gamma rhythms in human auditory cortex during musical beat processing. Ann NY Acad Sci, 1169, 89-92. 
530 Giroud, J., Trébuchon, A., Schön, D., Marquis, P., Liegeois-Chauvel, C., Poeppel, D., \&

531 Morillon, B. (2020). Asymmetric sampling in human auditory cortex reveals spectral

532 processing hierarchy. PLOS Biol, 18(3), e3000207.

533 Goswami, U. (2011). A temporal sampling framework for developmental dyslexia. Trends Cogn

$534 \quad$ Sci, 15, 3-10.

535 Goswami, U. (2015). Sensory theories of developmental dyslexia: Three challenges for research.

$536 \quad$ Nat R Neurosci, 16, 43-54.

537 Goswami, U. (2019). A neural oscillations perspective on phonological development and

538 phonological processing in developmental dyslexia. Lang Linguist Compass, 13, e12328.

539 Goswami, U., Mead, N., Fosker, T., Huss, M., Barnes, L., \& Leong, V. (2013). Impaired

540 perception of syllable stress in children with dyslexia: A longitudinal study. J Mem Lang,

$541 \quad 69,1-17$.

542 Goswami, U., Thomson, J., Richardson, U., Stainthorp, R., Hughes, D., Rosen, S., \& Scott, S. K.

543 (2002). Amplitude envelope onsets and developmental dyslexia: A new hypothesis. P

$544 \quad$ Natl Acad Sci USA, 99(16), 10911-10916.

545 Goswami, U., Wang, H.- L., Cruz, A., Fosker, T., Mead, N., \& Huss, M. (2011).

546 Language- universal sensory deficits in developmental dyslexia: English, Spanish and

$547 \quad$ Chinese. J Cognitive Neurosci, 23, 325-337.

548 Grahn, J. A. (2012). Neural mechanisms of rhythm perception: current findings and future 549 perspectives. Top Cogn Sci, 4, 585-606.

550 Grahn, J. A., \& Brett, M. (2009). Impairment of beat-based rhythm discrimination in Parkinson's 551 disease. Cortex, 45(1), 54-61. 
552 Habib, M., Lardy, C., Desiles, T., Commeiras, C., Chobert, J., \& Besson, M. (2016). Music and 553 dyslexia: A new musical training method to improve reading and related disorders. Front $554 \quad$ Psychol, 7, 26.

555 Haegens, S., \& Zion Golumbic, E. (2018). Rhythmic facilitation of sensory processing: A critical 556

Hämäläinen, J. A., Salminen, H. K., \& Leppänen, P. H. (2013). Basic auditory processing deficits in dyslexia: Systematic review of the behavioral and event-related potential/field evidence. J Learn Disabil, 46, 413-427.

Huss, M., Verney, J. P., Fosker, T., Mead, N., \& Goswami, U. (2011). Music, rhythm, rise time perception and developmental dyslexia: Perception of musical meter predicts reading and phonology. Cortex, 47(6), 674

Iversen, J., Repp, B., \& Patel, A. (2009). Top-down control of rhythm perception modulates early auditory responses. Ann NY Acad Sci, 1169(1), 58-73.

King, W. M., Lombardino, L. J., Crandell, C. C., \& Leonard, C. M. (2003). Comorbid auditory processing disorder in developmental dyslexia. Ear Hearing, 24(5), 448-456.

Kong, Y. Y., Mullangi, A., \& Ding, N. (2014). Differential modulation of auditory responses to attended and unattended speech in different listening conditions. Hearing Res, 316, 73-81.

Kotz, S. A., \& Schwartze, M. (2010). Cortical speech processing unplugged: A timely subcortico-cortical framework. Trends Cognitive Sci, 14, 392-399.

Law, J. M., Wouters, J. \& Ghesquiere, P. (2015). Morphological Awareness and Its Role in Compensation in Adults with Dyslexia. Dyslexia, 21, 254-72. 
573 Leong, V., \& Goswami, U. (2014). Impaired extraction of speech rhythm from temporal

574 modulation patterns in speech in developmental dyslexia. Front in Human Neurosci, 8, 57596.

576 Leong, V., Hämäläinen, J., Soltész, F., \& Goswami, U. (2011). Rise time perception and 577 detection of syllable stress in adults with developmental dyslexia. J Mem Lang, 64(1),

579 Luck, S. J. (2014). An introduction to the event-related potential technique. MIT press.

580 Luo, C., \& Ding, N. (2020). Cortical encoding of acoustic and linguistic rhythms in spoken $581 \quad$ narratives. eLife, 9, e60433.

582 Megnin- Viggars, O., \& Goswami, U. (2013). Audiovisual perception of noise vocoded speech 583 in dyslexic and nondyslexic adults: The role of low- frequency visual modulations. Brain Lang, 124(2), 165-173.

Merchant, H., Grahn, J., Trainor, L., Rohrmeier, M., \& Fitch, W. T. (2015). Finding the beat: a neural perspective across humans and non-human primates. Phil T R Soc B, 370, 20140093.

Molinaro, N., Lizarazu, M., Lallier, M., Bourguignon, M., \& Carreiras, M. (2016). Out- of- synchrony speech entrainment in developmental dyslexia. Hum Brain Mapp, $37,2767-2783$.

Morillon, B., \& Baillet, S. (2017). Motor origin of temporal predictions in auditory attention. Proceedings of the National Academy of Sciences, 114(42), E8913-E8921. perception and dyslexia: Evidence from French. NeuroReport, 15, 1255-1259. 
Näätänen, R., \& Picton, T. (1987). The N1 wave of the human electric and magnetic response to sound: a review and an analysis of the component structure. Psychophysiology, 24(4), 375425 .

Overy, K. (2000). Dyslexia, temporal processing and music: The potential of music as an early learning aid for dyslexic children. Psychol of Music, 28, 218-229.

Penolazzi, B., Spironelli, C., Vio, C., \& Angrilli, A. (2010). Brain plasticity in developmental dyslexia after phonological treatment: A beta EEG band study. Behavl Brain Res, 209, 179-182.

Power, A. J., Colling, L. J., Mead, N., Barnes, L., \& Goswami, U. (2016). Neural encoding of the speech envelope by children with developmental dyslexia. Brain Lang, 160, 1-10.

Power, A. J., Mead, N., Barnes, L., \& Goswami, U. (2013). Neural entrainment to rhythmic speech in children with developmental dyslexia. Front Human Neurosci, 7, 777.

Przybylski, L., Bedoin, N., Krifi-Papoz, S., Herbillon, V., Roch, D., Léculier, L., Kotz, S. A., \& Tillmann, B. (2013). Rhythmic auditory stimulation influences syntactic processing in children with developmental language disorders. Neuropsychology, 27(1), 121-131.

Schön, D., \& Tillmann, B. (2015). Short- and long-term rhythmic interventions: Perspectives for language rehabilitation. Ann NY Acad Sci, 1337, 32-39.

Sharma, M., Purdy, S. C., \& Kelly, A. S. (2009). Comorbidity of auditory processing, language, and reading disorders. J Speech Lang Hearing Res, 52, 706-722

Shaywitz, S. E., Shaywitz, B. A., Fulbright, R. K., Skudlarski, P., Mencl, W. E., Constable, R. T., Pugh, K. R., Holahan, J. M., Marchione, K. E., Fletcher, J. M. \& Lyon, G. R. (2003). Neural systems for compensation and persistence: young adult outcome of childhood reading disability. Biol Psychiat, 54, 25-33. 
618 Siegel, L. S. (2006). Perspectives on dyslexia. Paed Child Health, 11(9), 581-587.

619 Snyder, J. S., \& Large, E. W. (2005). Gamma-band activity reflects the metric structure of 620 rhythmic tone sequences. Cogn Brain Res, 24, 117-126.

621 Spironelli, C., Penolazzi, B., \& Angrilli, A. (2008). Dysfunctional hemispheric asymmetry of 622 theta and beta EEG activity during linguistic tasks in developmental dyslexia. Biol 623 Psychol, 77, 123-131.

624 Stefanics, G., Fosker, T., Huss, M., Mead, N., Szucs, D., \& Goswami, U. (2011). Auditory 625 sensory deficits in developmental dyslexia: a longitudinal ERP study. NeuroImage, 57, $626 \quad 723-732$.

627 te Woerd, E. S., Oostenveld, R., de Lange, F. P., \& Praamstra, P. (2017). Impaired auditory-to628 motor entrainment in Parkinson's disease. J Neurophysiol, 117, 1853-1864.

629 te Woerd, E., Oostenveld, R., de Lange, F., and Praamstra, P. (2018). Entrainment for attentional 630 selection in Parkinson's disease. Cortex, 166-178.

631 Thomson, J. M., \& Goswami, U. (2008). Rhythmic processing in children with developmental 632 dyslexia: Auditory and motor rhythms link to reading and spelling. J Physiol-Paris, $633 \quad 102(1-3), 120-129$.

634 Thomson, J. M., Fryer, B., Maltby, J. \& Goswami, U. (2006). Auditory and motor rhythm 635 awareness in adults with dyslexia. J Res Read, 29, 334-348.

636 Thomson, J. M., Leong, V., \& Goswami, U. (2013) Auditory processing interventions and 637 developmental dyslexia: A comparison of phonemic and rhythmic approaches. Read $638 \quad$ Writ, 26,139-161.

639 Trainor, L.J., Chang, A., Cairney, J., Li, Y.C. (2018). Is auditory perceptual timing a core deficit 640 of developmental coordination disorder? Ann NY Acad Sci, 1423, 30-39 
641 Van Hirtum, T., Ghesquière, P., \& Wouters, J. (2019). Atypical neural processing of rise time by 642 adults with dyslexia. Cortex, 113, 128-140.

643 Wieland, E. A., McAuley, J. D., Dilley, L. C., \& Chang, S. E. (2015). Evidence for a rhythm 644 perception deficit in children who stutter. Brain Lang, 144, 26-34.

645 Wolff, P. H., Michel, G. F., Ovrut, M., \& Drake, C. (1990). Rate and timing precision of motor 646 coordination in developmental dyslexia. Dev Psychol, 26, 349.

647 Woodruff Carr, K., White-Schwoch T., \& Tiernay A. T. (2014). Beat synchronization predicts 648 neural speech encoding and reading readiness in preschoolers. P Natl Acad Sci USA, $649 \quad 111(40), 14559-14564$.

650 


\section{Figure Legends}

652 Figure 1 . The beta $(15-25 \mathrm{~Hz})$ power at bilateral auditory cortices. (A) The beta power time 653 series are presented as the mean \pm standard error across typical (control) and dyslexic

654 participants. (B) The spectra of beta power fluctuation (note that this is not the spectra of the raw 655 EEG waveform). (C) The phases of beta power time series at $2 \mathrm{~Hz}$. Each blue arrow represents 656 the phase angle of beta power for a single participant; the phase angles and lengths of the red 657 arrows represent the group-averaged angle and consistency of the angle distribution, 658 respectively. The phase angles differed significantly between groups at the right auditory cortex. 659 (p: p-value; a.u.: arbitrary unit) 